\title{
Particle-in-Cell Simulations of High-Power THz Generator Based on the Collision of Strongly Focused Relativistic Electron Beams in Plasma
}

\author{
Vladimir Annenkov ${ }^{1, *(\mathbb{D})}$, Evgeny Berendeev ${ }^{2}(\mathbb{D})$, Evgeniia Volchok ${ }^{1,2} \mathbb{D}$ and Igor Timofeev $^{1}(\mathbb{D})$ \\ 1 Budker Institute of Nuclear Physics, SB RAS, 630090 Novosibirsk, Russia; E.P.Volchok@inp.nsk.su (E.V.); \\ I.V.Timofeev@inp.nsk.su (I.T.) \\ 2 Institute of Computational Mathematics and Mathematical Geophysics, SB RAS, 630090 Novosibirsk, Russia; \\ berendeev@sscc.ru \\ * Correspondence: v.v.annenkov@inp.nsk.su
}

Citation: Annenkov, V.; Berendeev,

E.; Volchok, E., Timofeev I.

Particle-in-Cell Simulations of

High-Power THz Generator Based on

the Collision of Strongly Focused

Relativistic Electron Beams in Plasma.

Photonics 2021, 8, 172. https://

doi.org/10.3390/photonics 8060172

Received: 10 April 2021

Accepted: 17 May 2021

Published: 21 May 2021

Publisher's Note: MDPI stays neutral with regard to jurisdictional claims in published maps and institutional affiliations.

Copyright: (c) 2021 by the authors. Licensee MDPI, Basel, Switzerland. This article is an open access article distributed under the terms and conditions of the Creative Commons Attribution (CC BY) license (https:// creativecommons.org/licenses/by/ $4.0 /)$.

\begin{abstract}
Based on particle-in-cell simulations, we propose to generate sub-nanosecond pulses of narrowband terahertz radiation with tens of MW power using unique properties of kiloampere relativistic $(2 \mathrm{MeV})$ electron beams produced by linear induction accelerators. Due to small emittance of such beams, they can be focused into millimeter and sub-millimeter spots comparable in sizes with the wavelength of $\mathrm{THz}$ radiation. If such a beam is injected into a plasma, it becomes unstable against the two-stream instability and excites plasma oscillations that can be converted to electromagnetic waves at the plasma frequency and its harmonics. It is shown that several radiation mechanisms with high efficiency of power conversion $(\sim 1 \%)$ come into play when the radial size of the beam-plasma system becomes comparable with the wavelength of the emitted waves.
\end{abstract}

Keywords: plasma; electromagnetic waves; terahertz generation; linear induction accelerators; particle-in-cell simulations

\section{Introduction}

In recent years, the efforts of many scientific groups have been aimed at creating radiation sources in the terahertz $(\mathrm{THz})$ frequency range $(0.3-10 \mathrm{THz})$. The reason for this is a large number of different applications of such radiation in science and technology (see reviews [1,2] and references therein). The leading role in generating narrowband $\mathrm{THz}$ radiation is presently played by large and expensive accelerating facilities such as free-electron lasers [3-5]. The generation of high-power narrowband $\mathrm{THz}$ radiation using more compact devices continues to be a challenge for several reasons. In particular, the conversion of laser radiation using nonlinear crystals [6-8] faces the problem of changing the properties and even destruction of a nonlinear medium when going to high powers. In the region of less than $1 \mathrm{THz}$, a lot of success has been achieved by devices based on vacuum electronics, for example, backward wave oscillators (BWO) [9]. For development in this area, breakthrough technological solutions are needed to create channels of a very small cross-section with a very high surface quality, without unnecessary impurities. In addition, it is also necessary to generate beams with very high currents and very small cross sections (cathodes, corresponding optics). The structural elements of such devices are often of the order of the wavelength, which can lead to overheating at high powers. Increasing the frequency in devices based on the use of magnetic fields often requires very high field values. In recent years, great progress in filling the $\mathrm{THz}$ gap has been made by gyrotrons [10-12]. However, an increase in the radiation frequency in such devices is associated with the need to use magnetic fields of tens of Tesla. In addition, transition to high frequencies in this case is accompanied by a significant decrease in maximum power and radiation efficiency. 
The problem of low thresholds for the destruction of a nonlinear medium is naturally overcome in plasma. Plasma allows to maintain electromagnetic oscillations of very large amplitude, the frequency of which is tied to the plasma density. If we ensure their conversion into electromagnetic radiation of the $\mathrm{THz}$ range, then, on the one hand, we will avoid the need to use superstrong magnetic fields to generate $\mathrm{THz}$ radiation, and on the other hand, we will be able to change the radiation frequency by simply changing the plasma density. Such advantages of plasma have stimulated the development of plasmabased THz radiation sources, most of which are based on laser-plasma systems [13-19].

A promising way in the development of powerful narrowband $\mathrm{THz}$ radiation sources is the use of kiloampere electron beams. The power of such beams reaches several gigawatts; therefore, conversion into radiation of even a percent of this power makes it possible to produce electromagnetic (EM) emission at the level of hundreds of MW. In addition, such beams have a sufficiently long duration to provide a narrow radiation spectrum. Among the $\mathrm{THz}$ generation schemes developed in this direction, it is worth noting a powerful planar FEL driven by a large-size sheet electron beam forming by the high-current accelerator «ELMI» with beam energy $1 \mathrm{MeV}$, current $7 \mathrm{kA}$ and duration $3 \mu \mathrm{s}[20,21]$. The possibility of constructing a free-electron maser based on high-current electron beams generated in linacs is also being considered [22,23].

Long-pulse electron beams can also be effectively used to generate high-power EM radiation. In experiments on the injection of a sub-relativistic electron beam into plasma at the GOL-3 facility (BINP SB RAS), an unexpectedly high level of electromagnetic emission has been observed when the beam diameter decreases to a size of the order of the radiation wavelength [24]. To explain the experimental results, the theory of the beam-driven plasma antenna $[25,26]$ has been proposed. Using this model, it has become possible not only to shed light on the main mechanism of generation of EM waves in these beamplasma experiments, but also to propose new schemes for highly efficient generation of EM radiation at harmonics of the plasma frequency in a plasma with a pre-modulated density $[27,28]$. At present, experiments on the generation of radiation by relativistic beams in a plasma with regular density inhomogeneities [29] are being carried out at the GOL-PET facility (BINP SB RAS). In addition to processes in a single beam-plasma system, a significant increase in the level of electromagnetic emission is possible in the presence of colliding electron beams in plasma. Significant enhancement of the second harmonic emission is observed not only in the case of beams that excite counterpropagating plasma oscillations with different transverse profiles of electrostatic potential [30], but also with a special selection of the parameters of the beam-plasma system, at which three-wave interactions between the most unstable plasma oscillations and electromagnetic waves are realized at the linear stage of the beam-plasma instability [31].

In this paper, using particle-in-cell (PIC) simulations, we study the possibility to generate high-power $\mathrm{THz}$ radiation in plasma by the counterstreaming electron beams with a few $\mathrm{kA}$ currents and relativistic energies $\sim 2 \mathrm{MeV}$ typical to the linear induction accelerators [32]. In Section 2, we describe our 2D3V axially symmetric PIC model with open boundary conditions allowing for the steady-state injection of electron beams into a plasma. In Section 3, simulation results on the injection of both a single beam and two oppositely propagating beams are presented. This section also discusses the methodology for processing simulation results and analyzes them. A summary of the results obtained is presented in Section 4.

\section{Numerical Model}

To simulate the problem, we use the PIC method in which distribution functions of real particles is replaced by the ones of model particles

$$
\tilde{f}(\mathbf{r}, \mathbf{v}, t)=\int f\left(\mathbf{r}^{\prime}, \mathbf{v}, t\right) S\left(\mathbf{r}, \mathbf{r}^{\prime}\right) d \mathbf{r}^{\prime}=\sum_{j} S\left(\mathbf{r}, \mathbf{r}_{j}(t)\right) \delta\left(\mathbf{v}-\mathbf{v}_{j}(t)\right) .
$$


Here, $\mathbf{r}_{j}, \mathbf{v}_{j}$ are the coordinate and velocity of the particle with the index $j$. Model particles have a spatial charge distribution characterized by the shape function $S\left(\mathbf{r}, \mathbf{r}_{j}\right)$

$$
\rho(\mathbf{r}, t)=\sum_{j} q_{j} S\left(\mathbf{r}, \mathbf{r}_{j}(t)\right),
$$

and interact with each other only through the computational grid thereby contributing to the charge and current density.

To solve the equations of particle motion, we use the scheme of Boris [33]. This is an explicit scheme in which the motion of a particle is split into separate motion in the electric field and in the magnetic field. Electric currents are calculated using the scheme of Esirkepov [34] adapted for the cylindrical geometry. In this scheme, at each step the charge density of the particle at the start and end points of movement is calculated by Formula (2) for a given form factor, and then used in the difference analogue of the continuity equation to obtain the current density. It automatically satisfies the difference continuity equation and, therefore, accurately fulfils the Gaussian difference law. The charge density of model particles are described by the fourth-order shape function:

$$
S\left(r, r^{\prime}\right)= \begin{cases}115 / 192-0.625 d^{2}+0.25 d^{4}, & d<h / 2 \\ \left(55+20 d-120 d^{2}+80 d^{3}-16 d^{4}\right) / 96, & h / 2 \leq d<3 h / 2 \\ (5-2 d)^{3} / 384, & 3 h / 2 \leq d<5 h / 2 \\ 0, & \text { otherwise. }\end{cases}
$$

Here, $d=\left|r-r_{j}\right|$ and $h$ is the grid step. We have chosen the fourth-order of the form factor in order to decrease numerical noise associated, among other things, with the numerical Cherenkov instability.

To solve Maxwell's equations, we use the finite-difference scheme (FDTD) proposed in [35], which provides the second order of accuracy in time and space. The problem is considered in the axially symmetric $r-z$ geometry, therefore the particles and grid cells are represented by uniform rings.

The computational domain contains a continuous plasma column bounded in the radial direction by vacuum (Figure 1). The dimensions of the area are chosen equal to $L_{z}=100 c / \omega_{p}$ and $L_{r}=32 c / \omega_{p}$, the grid and time steps are $h_{r}=h_{z}=0.02 c / \omega_{p}$ and $\tau=0.01 \omega_{p}^{-1}$. The plasma width is $L_{\text {plasma }}=20 c / \omega_{p}$. We use 49 model particles per cell for each particle species. In the $z$-direction, we set open boundary conditions described in [36]. Outside the area, we use the perfectly matched layers (PML) [37] in all directions to eliminate reflections of the produced EM waves.

We assume that the particles of the injected beams moving without the action of external forces would have a Gaussian distribution of density $n(x, y)$ and momenta $f\left(p_{x}, p_{y}\right)$ in the focal region $z=0$ :

$$
\begin{aligned}
n(x, y) & =\frac{n_{b}}{2 \pi \sigma_{r}^{2}} \exp \left(-\frac{x^{2}+y^{2}}{2 \sigma_{r}^{2}}\right), \\
f\left(p_{x}, p_{y}\right) & =\frac{1}{2 \pi \sigma_{p}^{2}} \exp \left(-\frac{p_{x}^{2}+p_{y}^{2}}{2 \sigma_{p}^{2}}\right) .
\end{aligned}
$$

The dispersions $\sigma_{r}, \sigma_{p}$ of these distributions determine the focal spot sizes and focusing angles. The quantity $n_{b}$ defines the beam density. Along the $z$-axis, beam particles have the same momentum $p_{z}$. Therefore, to simulate the injection of focusing beams, at each step, we first set the required distribution of particles at the focus in 3D3V space (for this purpose, it is sufficient to consider only one cell in the $z$-direction), after which we move the particles with a given momentum without external forces to the boundary at which injection will take place (Figure 2): 


$$
\begin{aligned}
n(x, y, z) & \rightarrow n^{\prime}\left(x^{\prime}, y^{\prime}, z^{\prime}\right): \\
x^{\prime} & =x-L p_{x} / p_{z}, \\
y^{\prime} & =y-L p_{y} / p_{z} \\
z^{\prime} & =z+L . \\
f\left(p_{x}, p_{y}, p_{z}\right) & \rightarrow f^{\prime}\left(p_{x}^{\prime}, p_{y}^{\prime}, p_{z}^{\prime}\right): \\
p_{x}^{\prime}=p_{x}, p_{y}^{\prime} & =p_{y}, p_{z}^{\prime}=p_{z} .
\end{aligned}
$$

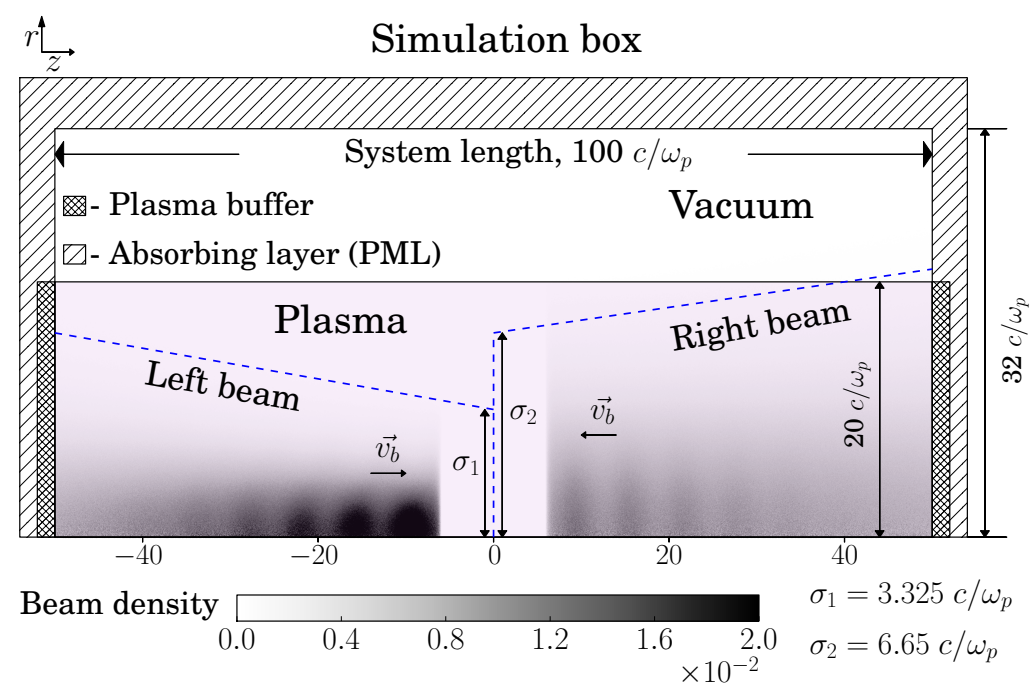

Figure 1. Layout of the computational domain.

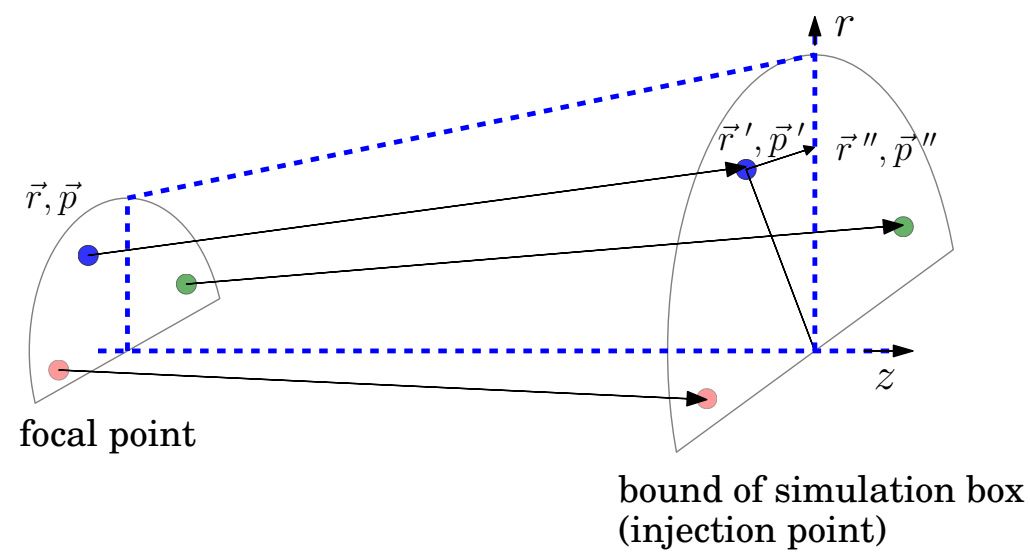

Figure 2. Coordinate transformation scheme for beam focusing.

Here, $L=L_{z} / 2$ is the distance from the focus to the border. Then we make the transition from 3D3V $\left(x^{\prime}, y^{\prime}, z^{\prime}, p_{x}^{\prime}, p_{y}^{\prime}, p_{z}^{\prime}\right)$ geometry to the axially symmetric system $\left(r^{\prime \prime}, z^{\prime \prime}, p_{r}^{\prime \prime}, p_{\phi}^{\prime \prime}, p_{z}^{\prime \prime}\right)$ and inject the resulting particles into the simulation region, having previously changed the direction of the pulse:

$$
\begin{aligned}
r^{\prime \prime} & =\sqrt{\left(x^{\prime}\right)^{2}+\left(y^{\prime}\right)^{2},} \\
p_{r}^{\prime \prime} & =\frac{x^{\prime}}{r^{\prime \prime}} p_{x}+\frac{y^{\prime}}{r^{\prime \prime}} p_{y}, \\
p_{\phi}^{\prime \prime} & =\frac{x^{\prime}}{r^{\prime \prime}} p_{y}-\frac{y^{\prime}}{r^{\prime \prime}} p_{x}, \\
p_{z}^{\prime \prime} & =p_{z}^{\prime}=p_{z} .
\end{aligned}
$$


The momentum spread $\sigma_{p}=\frac{R}{L} p_{z}$ is acquired due to focusing from the radius $R$ on the distance $L$. These dimensions are dictated by the intended experiment. The resulting radial distribution of beam density (Figure 3) is described by the formula:

$$
n(r)=n_{b} \exp \left(-\frac{r^{2}}{2 \sigma_{r}^{2}}\right)
$$

In our calculations, we consider two types of beams. The focal size of the first beam is $\sigma_{r}^{(1)} \equiv \sigma_{1}=3.325 c / \omega_{p}$, while its peak density is determined by the value $n_{b}^{(1)}=0.02 n_{0}$, where $n_{0}$ is the plasma density. We will call this beam as a "thin" one. The second beam ("wide") has $\sigma_{r}^{(2)} \equiv \sigma_{2}=6.65 c / \omega_{p}$ and $n_{b}^{(2)}=0.01 n_{0}$. The energy of beam particles is chosen equal to $2 \mathrm{MeV}$ which corresponds to the velocity $v_{b}=0.979 c(\gamma \approx 4.9)$. In this case, the thin beam is characterized by the power $P_{1}=3.665 \mathrm{GW}$ and current $I_{1}=2.9 \mathrm{kA}$. For the wide beam, we obtain $P_{2}=7.33 \mathrm{GW}$ and $I_{2}=5.8 \mathrm{kA}$.

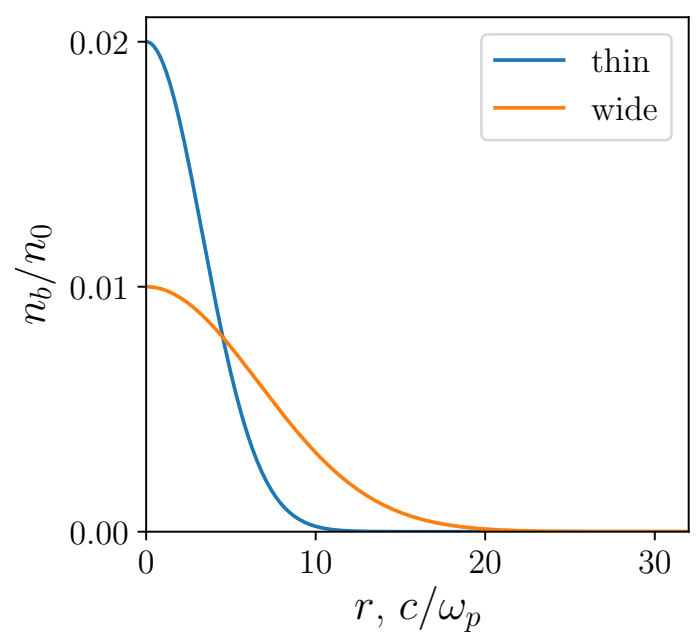

Figure 3. Radial profiles of the beams considered in the paper.

Further we will operate with dimensionless quantities. So all lengths will be measured in units of $c / \omega_{p}$, times in $\omega_{p}^{-1}$, electromagnetic fields in $m_{e} c \omega_{p} / e$. For the convenience of conversion into dimensional quantities, Figure 4 shows the dependence of these quantities, as well as the linear frequency of radiation at the harmonics of the plasma frequency, on the initial plasma density $n$. In particular, for the density $n_{0}=1.2512 \times 10^{15} \mathrm{~cm}^{-3}$ :

- the unit of length is $c / \omega_{p}=150 \mu \mathrm{m}$ and the focal spot-size is $\sigma_{2}=0.1 \mathrm{~cm}$;

- the radiation frequency at the third harmonic of plasma frequency corresponds to $f_{3}=\frac{3 \omega_{p}}{2 \pi} \approx 1 \mathrm{THz}$

- the unit of time $\omega_{p}^{-1}$ equals to $0.5 \mathrm{ps}$, and the total duration of a simulation run is $1000 \omega_{p}^{-1}=0.5 \mathrm{~ns}$;

- the typical dimensionless electric field amplitude in radiated waves constitutes $\widehat{E}_{\text {rad }}=0.005$ with the maximum $\widehat{E}_{\text {rad }}=0.01$, which in dimensional units correspond to $0.17 \mathrm{MV} / \mathrm{cm}$ and $0.34 \mathrm{MV} / \mathrm{cm}$. 


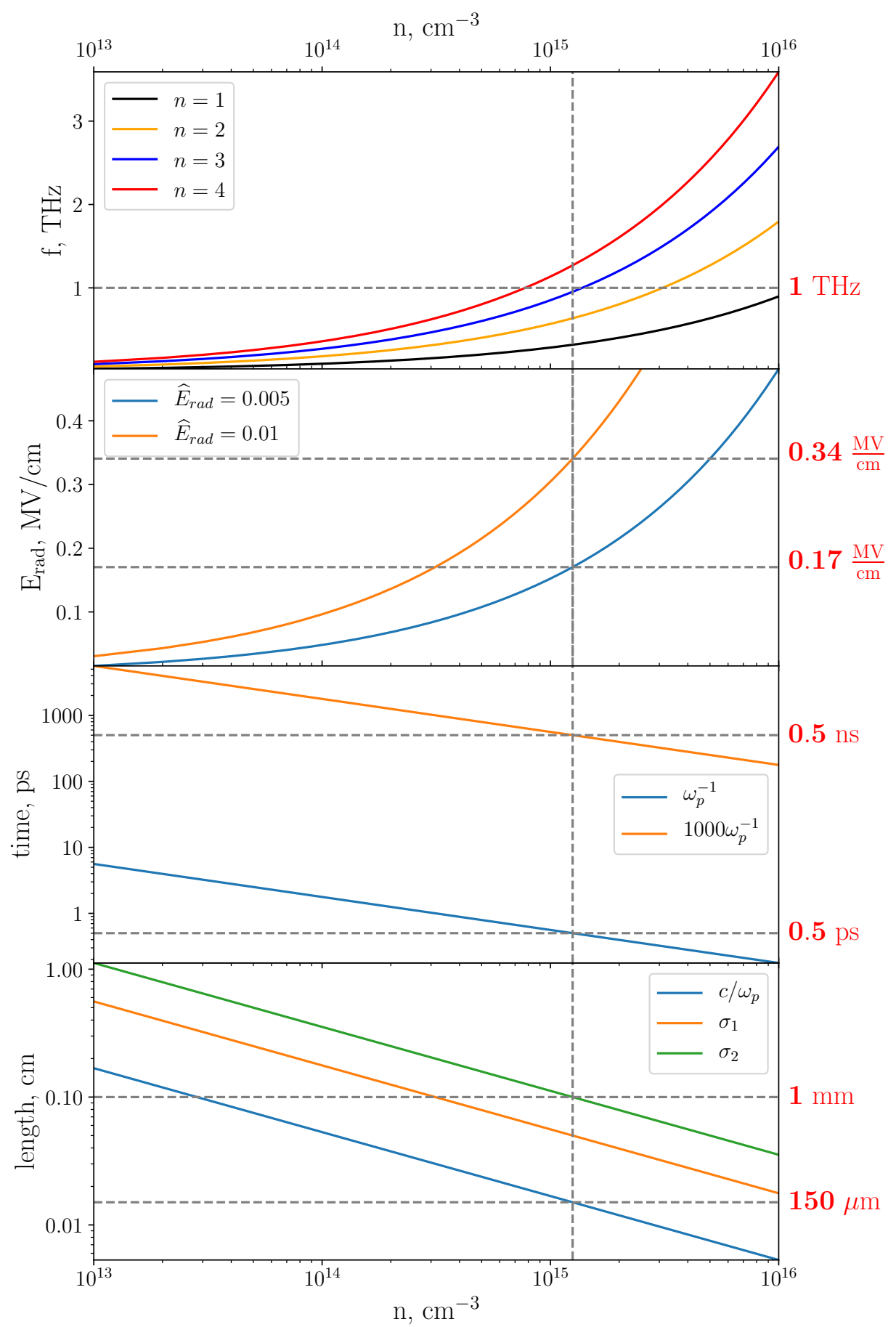

Figure 4. Correspondence between dimensional and dimensionless quantities. From top to bottom: the linear frequency of radiation at the plasma frequency harmonics; the characteristic amplitude of the radiation electric field; the unit of time $\omega_{p}^{-1}$ and total duration of simulations $1000 \omega_{p}^{-1}$; the unit of length $c / \omega_{p}$ and focal spot-sizes. The vertical dashed line corresponds to the plasma density $n_{0}=1.2512 \times 10^{15} \mathrm{~cm}^{-3}$.

\section{Simulation Results}

\subsection{Single Beam Injection}

Let us first consider the process of focusing a single beam into a spot of the width $\sigma_{1}=3.325 \mathrm{c} / \omega_{p}$ located in the center of the system. Figure 5 shows how the beam density and the corresponding profile of longitudinal electric field in the unstable plasma wave excited by the beam evolve in time. When the beam is injected, the intense two-stream 
instability develops in the focal region. This leads to the formation of a modulated beam structure already by the time $t \omega_{p}=150$. In this case, the characteristic distance between the forming bunches corresponds to the wavelength of the most unstable mode $\left(5.5 c / \omega_{p}\right)$. The location of beam focus in plasma, however, does not remain stationary and demonstrates the complicated dynamics.

Initially, the most intense excitation of plasma oscillations occurs at the geometrical focus of the beam, where it reaches the highest density. Later, however, one can see that the beam is focused closer to the injector $\left(t \omega_{p}=300\right)$. The reason for this refocusing is that a plasma compensates the charge of the beam which, in vacuum, tends to push particles from the axis via the radial electric field, but do not completely compensate the current which compresses the beam radially by its own azimuthal magnetic field. As a result, the beam turns out to be compressed to high densities before it reaches the focus that would be realized in a vacuum. Over time, the plasma wave becomes less regular and the bunch structure of the beam is less pronounced.
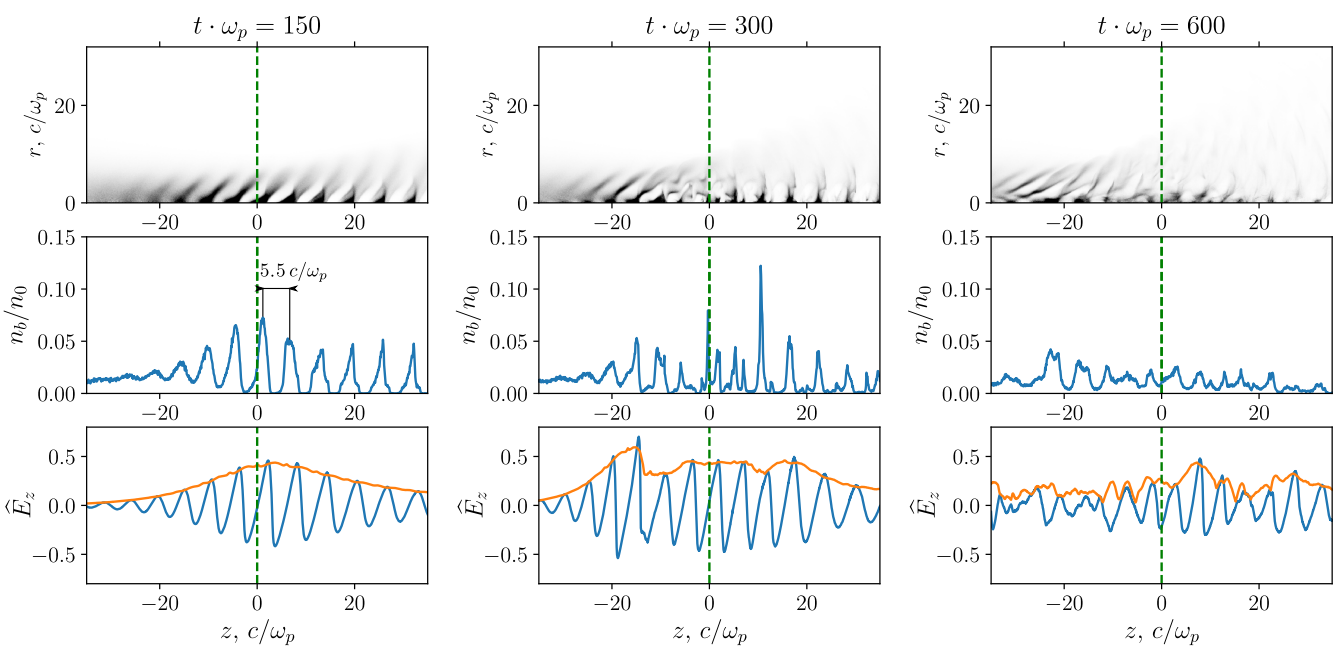

Figure 5. The beam density and profile of the beam-driven plasma wave in different moments of time. (Top): $n_{b}(r, z)$. (Middle): $n_{b}(z)$ near the axis. (Bottom): dimensionless amplitude of the longitudinal electric field $\widehat{E}_{z}(z)$ near the axis. The orange curve shows the envelope of the unstable wave packet. The green line markes the center of the system (the beam focal plane in vacuum).

Let us study spectral characteristics of beam-driven plasma oscillations in more detail. For this, we carry out a two-dimensional Fourier transform of the dependence of the longitudinal electric field $\widehat{E}_{z}(z, t)$ near the axis of the system on the longitudinal coordinate and time. Figure 6 shows the resulting spectrum. The dominant beam-driven mode has a longitudinal wavenumber $\left|k_{\|}\right|=\left|k_{0}\right| \approx 1.06 \omega_{p} / c$ and frequency $\omega_{0} \approx 0.96 \omega_{p}$. A large amplitude of plasma oscillations leads to the development of nonlinear processes accompanied by excitation of harmonic oscillations $\left(n \omega_{0}, n k_{0}\right)$. The frequency of plasma waves throughout the simulation remains constant with good accuracy, as evidenced by the narrow line-width in the Fourier spectrum. Contrary, the spread in wavenumbers is more significant.

Electromagnetic emission in this case is found to be very weak. We only observe negligible electromagnetic radiation at harmonics $2 \omega_{0}$ and $3 \omega_{0}$ with the efficiency of beamto-radiation power conversion at the level of $10^{-2} \%$ and $3 \times 10^{-3} \%$, respectively. The situation is not changed drastically even for mobile ions. In the case of a thin beam, one can expect the switching on of the highly efficient mechanism of plasma antenna when the ion density get an opportunity to be modulated in the longitudinal direction by largeamplitude plasma waves. But longitudinal modulation in the case of a single beam turns out to be a slower process than radial displacement of plasma from the beam region by the ponderomotive force of plasma oscillations. Emission can be enhanced only if we 
introduce either the guiding magnetic field eliminating the radial plasma dynamics or a counterpropagating beam that makes possible to generate radiation by the collision of different-size waves.

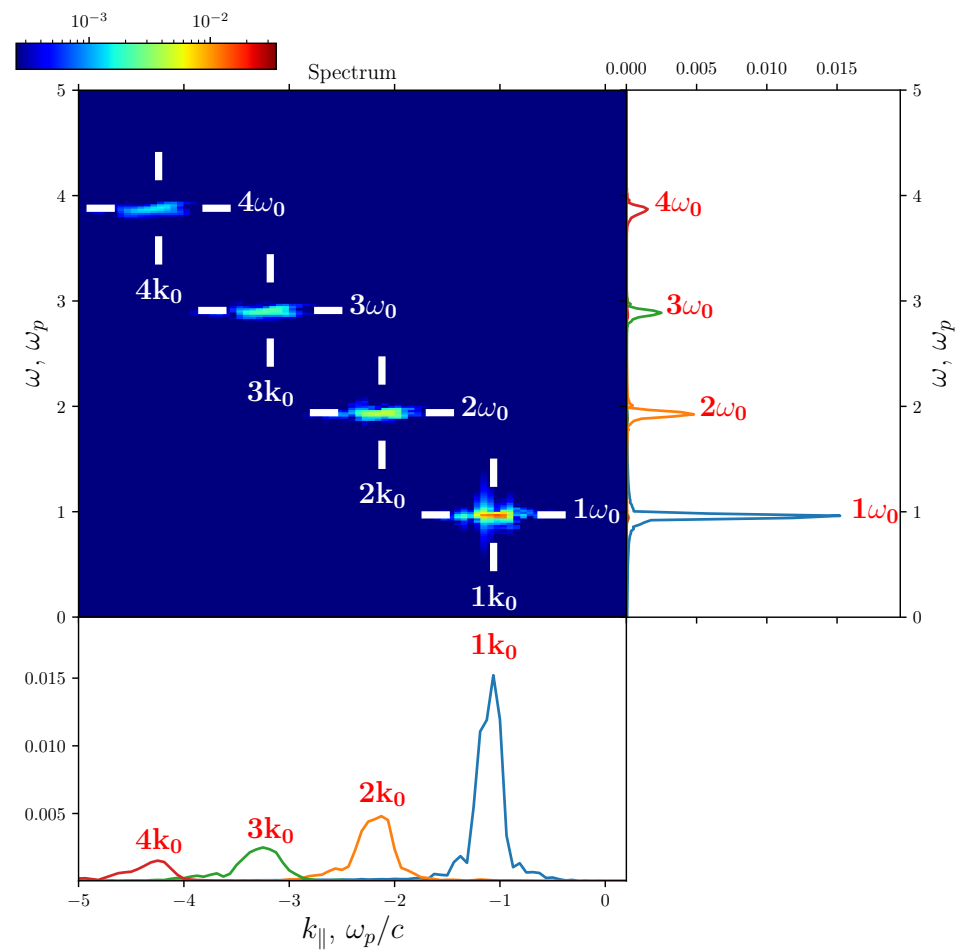

Figure 6. Fourier spectrum of plasma oscillations excited by a single beam.

Indeed, if the thin beam is guided by the strong magnetic field $B_{z}=1.1(\approx 12.5 \mathrm{~T}$ for the plasma density $n_{0}=1.25 \times 10^{15} \mathrm{~cm}^{-3}$ ), the maximum value of the total radiation power reaches $1.6 \%$ of the power injected by the beam (Figure 7 ). EM radiation appears when the ion density is modulated in the axial direction with the period appropriate for the antenna mechanism. It is also seen that the produced radiation is strongly dominated by the fundamental frequency $\omega_{0}$.
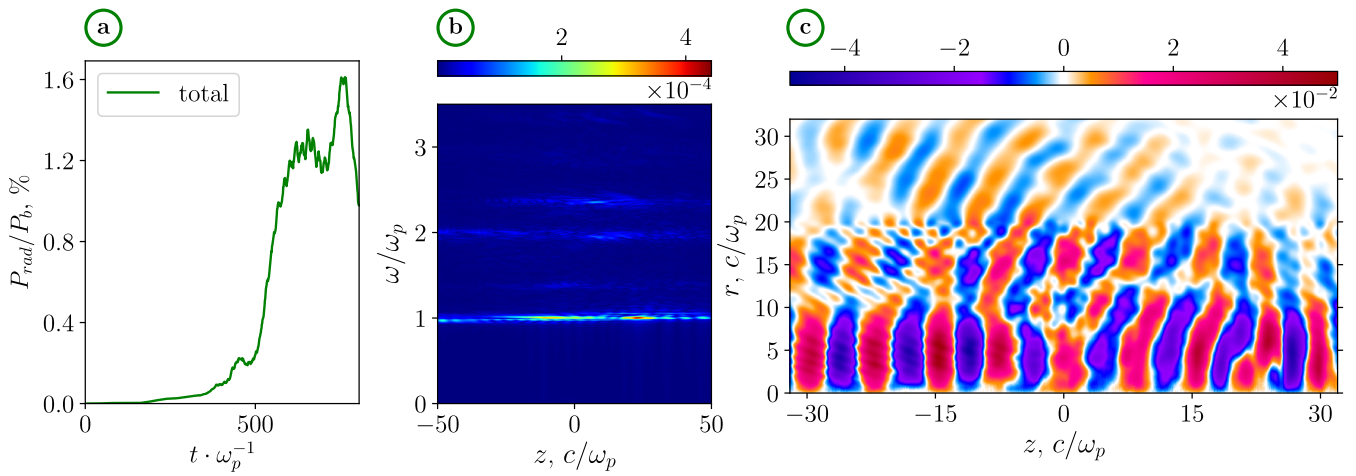

Figure 7. (a) total radiation efficiency at all frequencies; (b) radiation spectrum calculated at each point on the boundary of the computational domain; (c) electric field $E_{\phi}$ in the moment $t=580 \omega_{p}^{-1}$.

\subsection{Processing Technique of Radiation Signals}

Before proceeding to a discussion of simulation results obtained for the two-beam systems, let us consider the technique we use for processing the recorded radiation signals. Figure 8 (top) shows a part of the time dependence of a typical field signal $\widehat{E}_{z}$ recorded at one spatial point on the boundary of the computational domain. Due to the significant nonlinearity of the oscillations excited by the beams, the radiation contains not only the 
second, but also higher harmonics. In order to investigate their relative contributions separately, we use Fourier filtering. For each radiation signal, we carry out a direct Fourier transform and obtain its spectrum (Figure 8 on the left). Then we carry out the inverse Fourier transform separately for parts of the spectrum in the range $n \omega_{p} \pm 0.5$ (Figure 8 on the right). Figure A1 in the Appendix A shows the temporal dependences of the field amplitude $\widehat{E}_{z}$ at points on the boundary of the vacuum region for each radiation harmonic in each of the simulated two-beam systems. A similar procedure is carried out for the $\widehat{B}_{\phi}$ field. Knowing the fields of individual harmonics $\widehat{E}_{z}$ and $\widehat{B}_{\phi}$ on the boundary of the vacuum system, one can find their contribution to the normal component of the Poynting vector $\widehat{E}_{z} \cdot \widehat{B}_{\phi}$, from which we obtain the power of radiation leaving the system in the radial direction at a given harmonic (Figure 8 bottom). By integrating its value along the boundary of the vacuum region at each moment of time, we obtain the temporal dependence of the total power radiated in all directions. The ratio of the obtained value to the power of the injected beams will give us the efficiency of radiation generation.
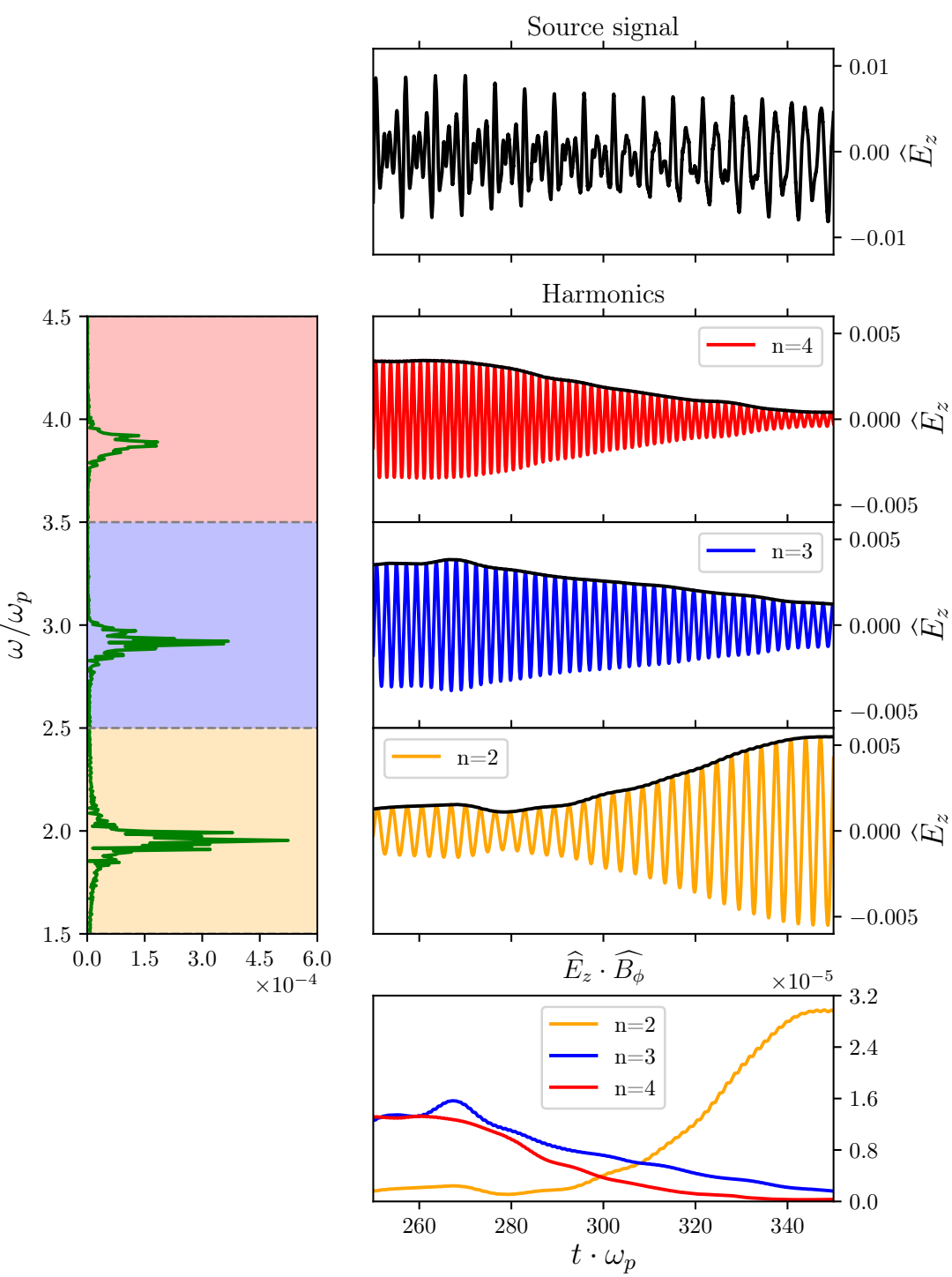

Figure 8. Processing technique of radiation signals. (Top): initial radiation signal (field $\widehat{E}_{z}$, recorded at one point on the boundary of the computational domain). (Left): Fourier spectrum of the initial signal. (Right): individual harmonics of the signal. (Bottom): temporal dependence of the radial component of the Poynting vector for each radiation harmonic. 


\subsection{Counter Injection of Two Symmetric Thin Beams}

Let us study the process of radiation generation in a plasma with two colliding beams. Let us first consider the case of two thin identical beams, as well as the case of asymmetric collision of thin and wide beams in a plasma with immobile (infinitely heavy) ions. Then, we estimate the effect of mobile hydrogen ions with a real mass on the radiation process in both cases.

Let two colliding electron beams be focused to the center of the system into spots with the same sizes $\sigma_{1}=\sigma_{2}=3.325 \mathrm{c} / \omega_{p}$. Figure 9 shows the spectrum of oscillations excited by the beams. In addition to oscillations $\left(n \omega_{0}, \pm n k_{0}\right)$ arising from each beam, oscillations at the combined frequencies and wavenumbers are observed in the spectrum.

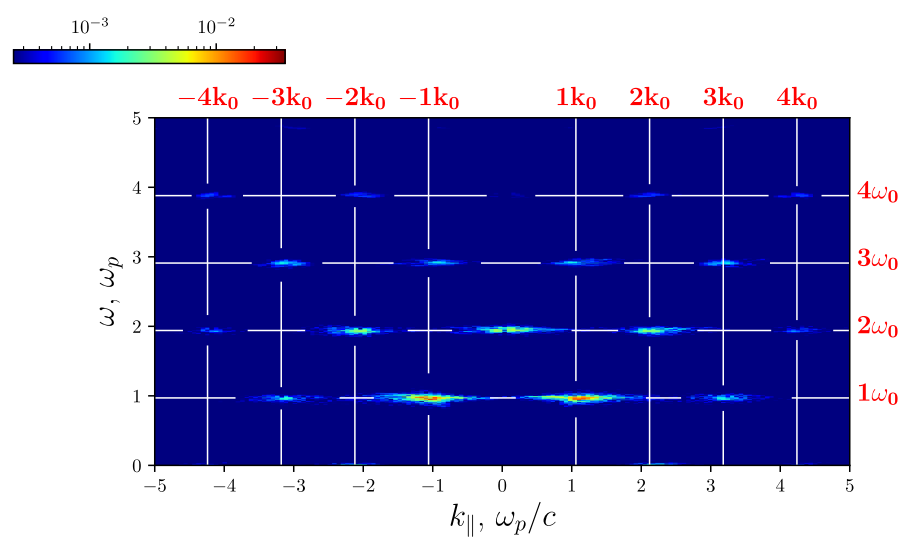

Figure 9. Fourier spectrum of plasma oscillations driven by counterstreaming thin beams in the case of immobile ions.

Resonance with vacuum EM waves can be achieved only for those plasma oscillations that travel along the plasma column with superluminal phase velocities. For this reason, neither the most unstable modes $\left(\omega_{0}, \pm k_{0}\right)$ directly excited by each of the beams at the Cherenkov resonance, nor their harmonics can be responsible for EM emission. Superluminal satellites in plasma can be excited only by nonlinear interaction of counterpropagating beam modes. For example, radiation at the second harmonic is generated due to the coalescence

$$
\left(\omega_{0}, k_{0}\right)+\left(\omega_{0},-k_{0}\right) \rightarrow\left(2 \omega_{0}, 0\right),
$$

while the third and forth harmonics arise in the processes:

$$
\begin{aligned}
& \left(\omega_{0}, \mp k_{0}\right)+\left(2 \omega_{0}, \pm 2 k_{0}\right) \rightarrow\left(3 \omega_{0}, \pm k_{0}\right), \\
& \left(\omega_{0}, \pm k_{0}\right)+\left(2 \omega_{0}, 0\right) \rightarrow\left(3 \omega_{0}, \pm k_{0}\right), \\
& \left(\omega_{0}, \mp k_{0}\right)+\left(3 \omega_{0}, \pm 3 k_{0}\right) \rightarrow\left(4 \omega_{0}, \pm 2 k_{0}\right), \\
& \left(\omega_{0}, \pm k_{0}\right)+\left(3 \omega_{0}, \pm k_{0}\right) \rightarrow\left(4 \omega_{0}, \pm 2 k_{0}\right), \\
& \left(2 \omega_{0}, \pm 2 k_{0}\right)+\left(2 \omega_{0}, 0\right) \rightarrow\left(4 \omega_{0}, \pm 2 k_{0}\right) .
\end{aligned}
$$

The longitudinal phase velocities of the oscillations involved in these nonlinear interactions are given in Table 1.

Table 1. Phase velocities of plasma oscillations $v_{p h} / c=\omega /\left|k_{\|}\right|$.

\begin{tabular}{lcccc}
\hline & $\mathbf{1} k_{0}$ & $\mathbf{2} k_{0}$ & $3 k_{0}$ & $4 k_{0}$ \\
\hline $1 \omega_{0}$ & 0.92 & & 0.3 & \\
$2 \omega_{0}$ & & 0.92 & & 0.46 \\
$3 \omega_{0}$ & 2.75 & & 0.92 & \\
$4 \omega_{0}$ & & 1.83 & & 0.92 \\
\hline
\end{tabular}


The process (17), however, degenerates if the dominant beam modes are excited by symmetric drivers and run along the beam axis. Indeed, the symmetry of the system in this case is so high that it leads to the vanishing of the electric current quadratic in the field. As shown in recent works $[15,30]$, the asymmetry necessary for the generation of radiation in this case can be provided due to differing profiles of the electrostatic potential in colliding waves $\Phi_{1}\left(\mathbf{r}_{\perp}\right) \neq \Phi_{2}\left(\mathbf{r}_{\perp}\right)$. The generating current then has the form

$$
\mathcal{J}_{\|}=\frac{k_{0}}{\omega_{0}^{3}}\left(\Phi_{1} \Delta_{\perp} \Phi_{2}-\Phi_{2} \Delta_{\perp} \Phi_{1}\right),
$$

and the radiation it produces is directed strictly across the axis of the system.

Knowing the frequency and longitudinal wavenumber of oscillations, as well as the dispersion law of electromagnetic oscillations in vacuum $\omega=k c$, one can find the angle at which radiation should be generated in the processes (18)-(22):

$$
\alpha=\arctan \frac{k_{\perp}}{k_{\|}} .
$$

Here, $k_{\perp}$ is the transverse wavenumber of emitted EM waves. For emission at the frequency $3 \omega_{0}$,

$$
k_{\perp}=\sqrt{9 \omega_{0}^{2}-k_{\|}^{2}} .
$$

Then the angle of radiation is

$$
\alpha_{3}=\arctan \frac{\sqrt{9 \omega_{0}^{2}-k_{\|}^{2}}}{k_{\|}} .
$$

Similarly, we obtain the angle of the fourth harmonic radiation:

$$
\alpha_{4}=\arctan \frac{\sqrt{16 \omega_{0}^{2}-4 k_{\|}^{2}}}{2 k_{\|}} .
$$

The frequencies of the plasma oscillations responsible for the radiation are determined unambiguously, while the wavenumbers have a certain scatter, which should lead to an uncertainty in the radiation angle. Further, based on the results shown in Figure 6, we will consider the longitudinal wavenumber $k_{\|}$to be in the range $[0.95 ; 1.2]$. Thus, the third and forth harmonic radiations are predicted to be emitted at angles

$$
\alpha_{3} \in\left[65^{\circ} ; 71^{\circ}\right], \quad \alpha_{4} \in\left[51^{\circ} ; 60^{\circ}\right] .
$$

Let us focus on the radiation processes observed in PIC simulations in such a beamplasma system. Figure 10 shows simulation results in different moments of time. At the initial stage $\left(t=200 \omega_{p}^{-1}\right)$, the particles of both beams are focused into the center where the largest amplitude of plasma oscillations is achieved. It can be seen that radiation is generated in the center of the system at the third and fourth harmonics. The radiation angles in this case correspond to those theoretically predicted in (27). In the given moment of time, the local transverse profiles of plasma oscillations excited by each beam coincide with good accuracy over the entire length of the system, and radiation at the second harmonic is not observed. Later $\left(t=262 \omega_{p}^{-1}\right)$, the beams are refocused in the same manner as discussed in the single beam case and the regions of intense relaxation for each of the beams are shifted towards their own injectors. Two spaced regions with a high amplitude of plasma oscillations are formed. Each region becomes a source of radiation at the third and fourth harmonics. By the time moment $t=345 \omega_{p}^{-1}$, the relaxation regions of the beams are significantly removed from the center of the system. In each given region, 
the counterpropagating beam-driven waves have different transverse structures. This is a necessary condition for switching on the mechanism of radiation generation near the second harmonic of the plasma frequency (23). The appearance of such radiation is clearly observed in Figure 10. In the center of the system, a clear zero of the radiation field is visible, since this mechanism does not work here due to the symmetry of the plasma waves. In the main relaxation zone of each beam, the excitation of counterpropagating oscillations by the opposite beam is reduced. This leads to a relative decrease in the amplitude of nonlinear harmonic oscillations in plasma and to a decrease in the radiation power at frequencies $3 \omega_{0}$ and, in particular, $4 \omega_{0}$.
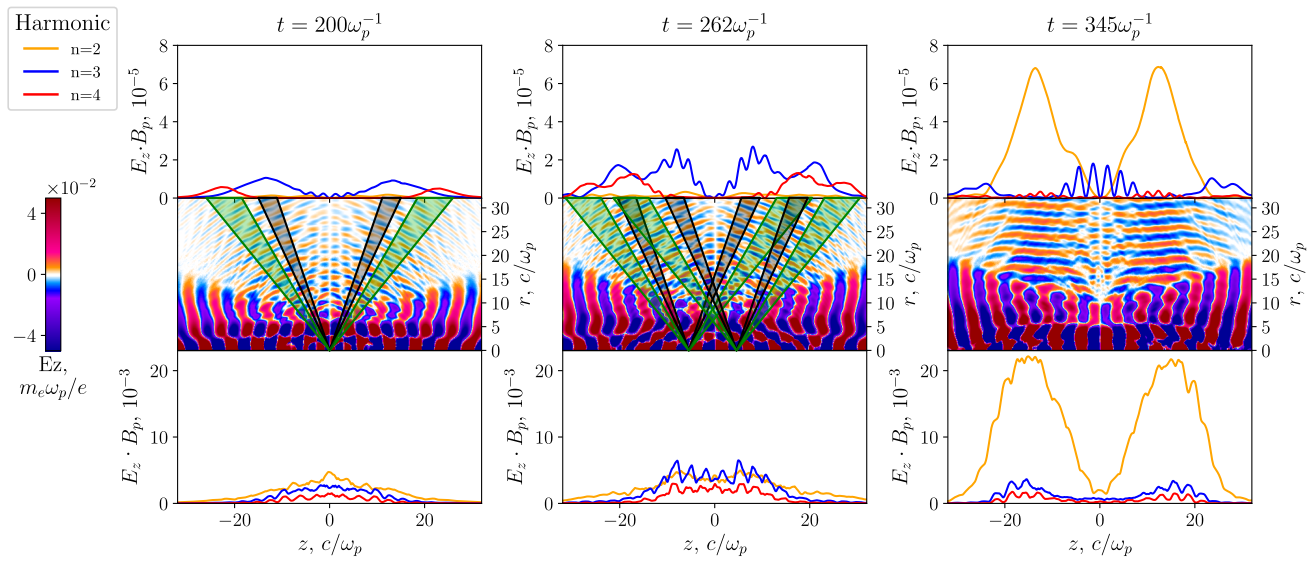

Figure 10. Results of PIC simulations in different moments of time for the collision of two identical thin beams. The radial component of the Poynting vector $E_{z} \cdot B_{\phi}$ on the top boundary in vacuum (top row) and near the axis of the system inside the plasma (bottom row). The maps of electric field $\widehat{E}_{z}(z, r)$ (middle row). The green cone shows theoretical predictions for the direction of $4 \omega_{0}$-radiation and the black cone-for $3 \omega_{0}$-radiation.

Let us analyze the frequency spectrum of the generated radiation. Figure 11 shows the Fourier spectrum of radiation detected at the boundary of the vacuum region and the spatial distribution of the energy emitted at each harmonic during the entire simulation time. It is seen that each harmonic is characterized by a narrow spectral line $(\Delta \omega / \omega \sim 1 \%)$, and the dominant role is played by the second harmonic radiation. The spatial profile of radiation intensity at the frequency $4 \omega_{0}$, integrated over the entire simulation time, corresponds to the early stage in Figure 10. The fourth harmonic emission is the result of high-order nonlinearity which requires high amplitude of plasma oscillations and is efficiently realized at the early stages of the process, when the relaxation regions of each beam are well overlapped. The spatial distribution of the third harmonic is more uniform, while the second harmonic radiation demonstrates the pronounced peaks from the newly formed foci of colliding beams.

Note that distribution functions of beams and plasma in numerical simulations are described using a finite number of model particles. To model a finite temperature, one should set a scatter in particle momenta using a random number generator. For these reasons, all numerical simulations, even with the same macro parameters, may differ in details from each other. Since the beam-plasma instability grows from these noise fields, dynamics of counterpropagating unstable waves is not fully symmetric. It explains the asymmetric spatial distribution for the second harmonic emission that is observed in Figure 11. The issue of repeatability for numerical simulations of beam-plasma interaction has been discussed in more details in [31]. 


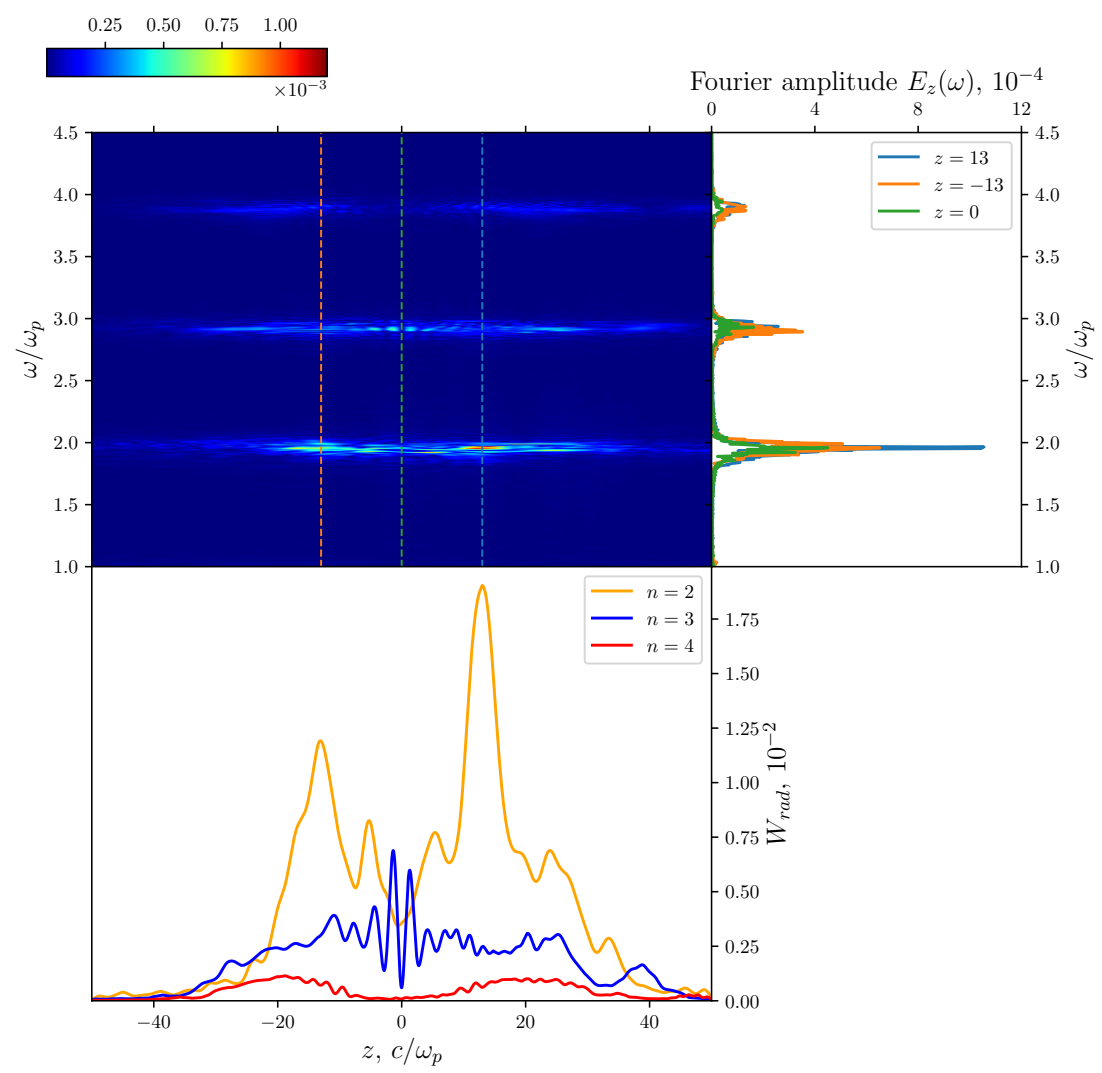

Figure 11. Spectrum (top) and spatial distribution of radiated energy (bottom) for the collision of identical beams. On the right: spectra along the lines with corresponding colors.

\subsection{Counter Injection of Different-Size Beams}

Let us now consider the generation of radiation by colliding beams focused into different-size spots. The size of the left beam remains equal to $\sigma_{1}=3.325 \mathrm{c} / \omega_{p}$, while the right beam is chosen twice wider $\sigma_{2}=6.65 \mathrm{c} / \omega_{p}$. Results of simulations of such a system in different moments of time are presented in Figure 12. In this case, colliding beams excite plasma oscillations with different transverse structures from the very beginning; therefore, in contrast to the case of identical thin beams, the second harmonic emission here is observed immediately after the development of the two-stream instability. EM waves at higher harmonics are also visible at the initial stage, but they are mainly emitted along the direction of thin beam propagation. It is explained by the dominance at this stage of a plasma wave excited by the thin beam. Further development of two-stream instability leads to an increase in the amplitude of plasma waves. The radiation at high harmonics is amplified and begins to significantly exceed the fraction of radiation at the second harmonic. It is interesting that, by the later time moment $t=580 \omega_{p}^{-1}$, this radiation changes direction and begins to significantly dominate over other emission processes.

Figure 13 shows the spectrum of the generated EM radiation and the spatial distribution of its energy accumulated during the entire simulation time. According to the theory, the radiation at the frequency $2 \omega_{0}$ was expected to dominate due to the interaction of counterpropagating plasma oscillations with different transverse structures. However, such radiation was effectively generated only at the initial stage of interaction. At later times, the greatest contribution to the total radiation power is made by the third harmonic. 

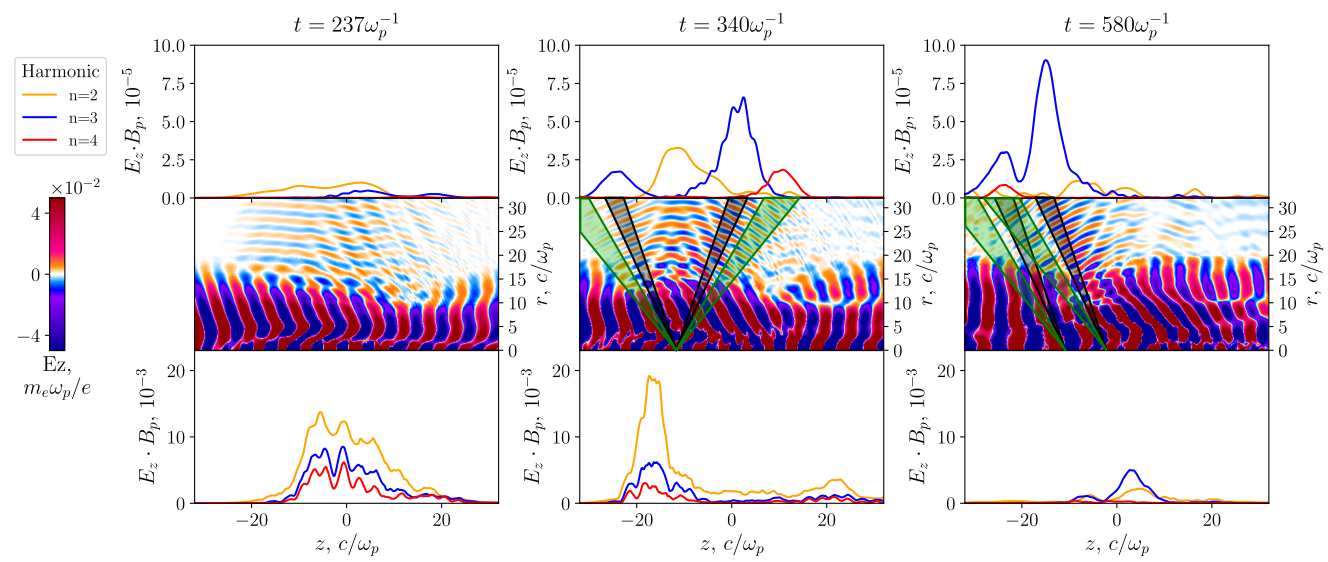

Figure 12. Results of PIC simulations for the collision of different-size beams.

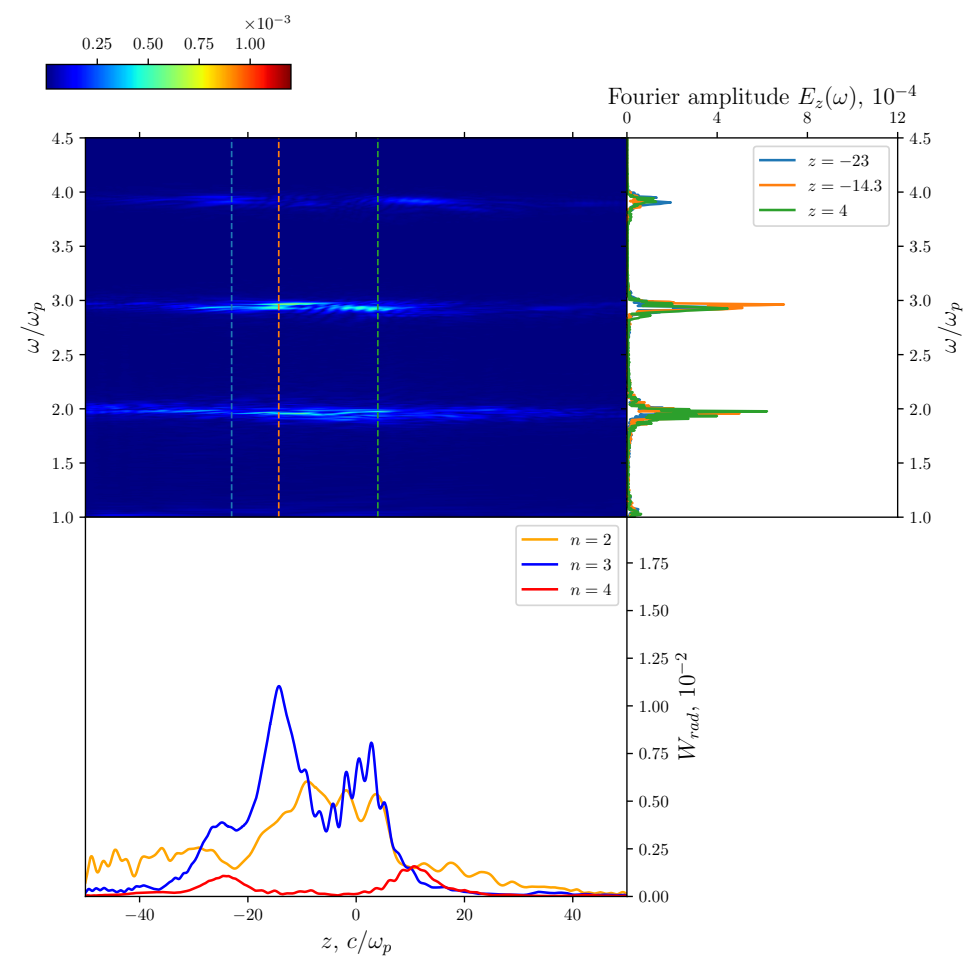

Figure 13. Spectrum (top) and spatial distribution of radiated energy (bottom) for the collision of different-size beams. On the right: spectra along the lines with corresponding colors.

\subsection{Ion Effects}

Let us find out how the parameters of EM emission at the plasma frequency harmonics will change if hydrogen ions with a real mass $\left(m_{i}=1836 m_{e}\right)$ are used in the scheme with two thin beams. Results of PIC simulations in this case are presented in Figure 14. It can be seen from the ion density maps that, in addition to the displacement of the plasma along the radius, the formation of longitudinal modulation of the plasma density with the wavenumber $2 k_{0}$ is also observed. In contrast to the case of a single beam, the longitudinal modulation in the system of counterstreaming beams grows much faster due to the fact that superposition of colliding beam-driven oscillations form a standing plasma wave. The ponderomotive force in such a wave is modulated in space, therefore, by displacing electrons from the nodes of the wave and creating an uncompensated charge, it leads to modulation of the ion density. At the early stages, when the ion density perturbation can be considered as a small one, its presence should enhance the radiation at the second harmonic, since each beam-driven wave is able to radiate by the additional mechanism 
of the plasma antenna $[26,27,38]$. However, already by the time $t=220 \omega_{p}^{-1}$, the ion modulation becomes so deep that it significantly weakens the beam instability [39], leading to a significant decrease in the radiation intensity at all harmonics (Figure 15).

Thus, the duration of the radiation during counter-beam injection is limited to the times at which the ion dynamics becomes significant. For plasma densities $n_{0} \approx 1.2 \times 10^{15} \mathrm{~cm}^{-3}$ this duration is a fraction of a nanosecond. With increasing ion mass (in this work, we have considered the lightest ions-hydrogen nuclei), the duration of radiation can be increased by increasing the duration of beam injections.
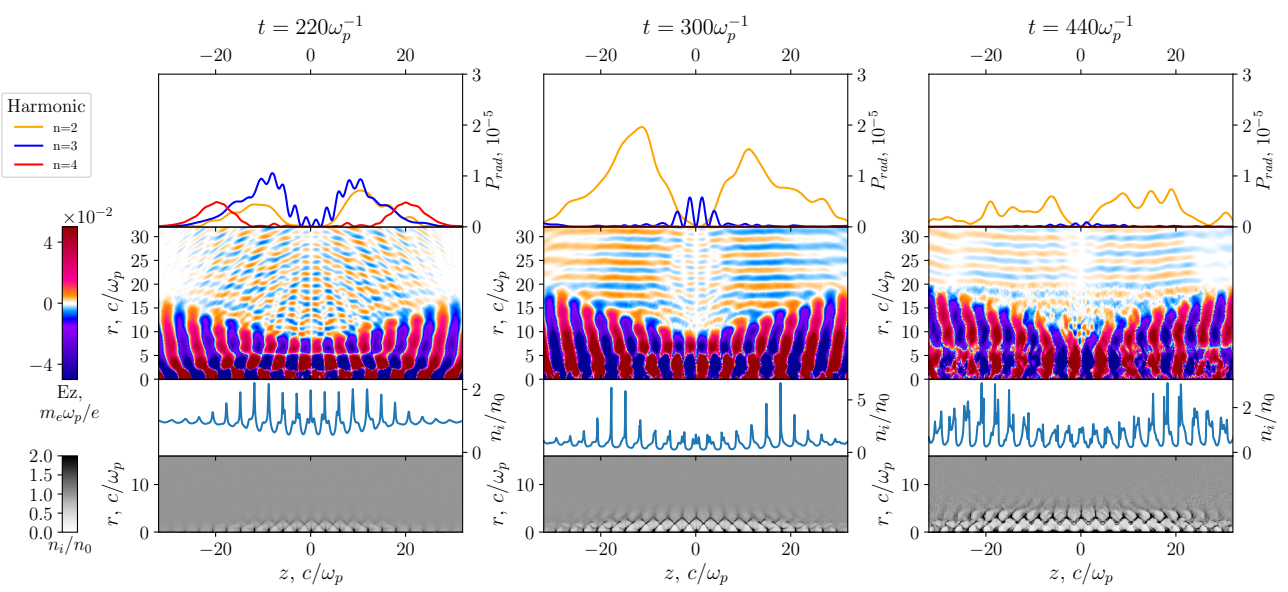

Figure 14. Results of PIC simulations accounting for ion dynamics in the case of two symmetric thin beams. The radial component of the Poynting vector $E_{z} \cdot B_{\phi}$ on the top boundary of the vacuum region (top row). The maps of electric field $\widehat{E}_{z}(z, r)$ (middle row). Ion density $n_{i}(z) / n_{0}$ on the axis of the system and inside the whole plasma column $n_{i}(r, z) / n_{0}$ (bottom row).

$10^{-3} \quad 10^{-2}$

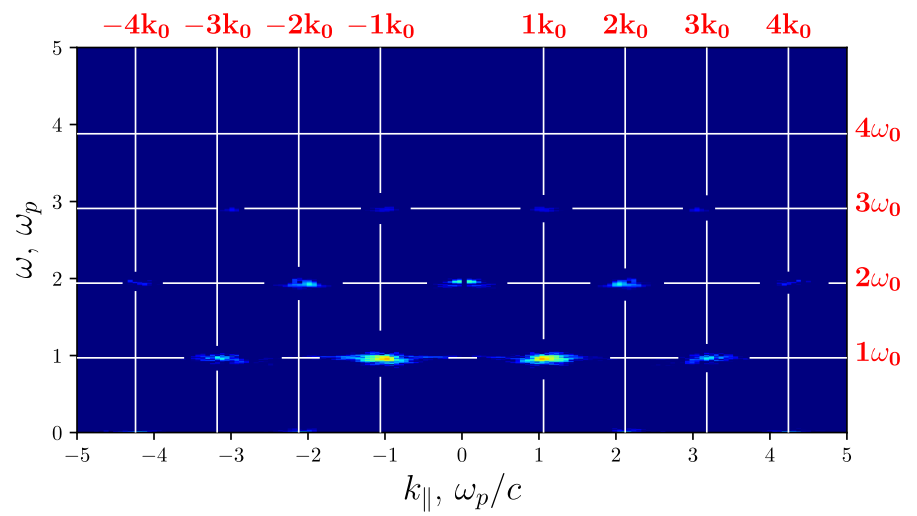

Figure 15. Fourier spectrum of plasma oscillations excited by symmetric thin beams in the case of mobile ions.

\subsection{Radiation Efficiency}

Let us estimate quantitatively the efficiency of THz generation $\eta=P_{r a d} / P_{b}$ in the system considered. Figure 16 shows what fraction of the total power injected with the beams $P_{b}$ is converted to the radiation power $P_{\text {rad }}$ at each harmonic for all regimes discussed in the work. The power of the thin beam reaches the value $P_{1}=3.67 \mathrm{GW}$ and the power of the wide beam $-P_{2}=7.33 \mathrm{GW}$. In the scheme of a single beam, efficient radiation can be generated only if the focused beam is guided by a strong magnetic field (Figure 7). In this case, the magnetic field prevents the radial dynamics of a plasma, which creates favorable conditions for the antenna mechanism at the stage of axial plasma density modulation. The maximum efficiency of beam-to-radiation power conversion reaches $1.6 \%$ and the spectrum of produced radiation is dominated by the fundamental frequency $\omega_{0}$. 


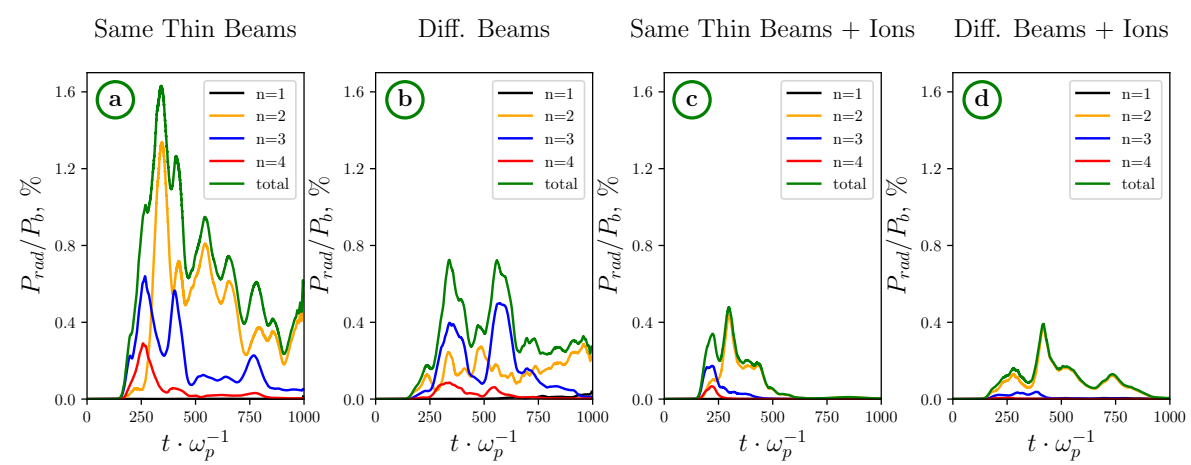

Figure 16. Efficiency of beam-to-radiation power conversion as a function of time for two-beam simulations. (a) same beams with $\sigma_{1}=\sigma_{2}=3.325 \mathrm{c} / \omega_{p} ;(\mathbf{b})$ different beams with $\sigma_{1}=3.325 \mathrm{c} / \omega_{p}$ and $\sigma_{2}=6.65 \mathrm{c} / \omega_{p} ;(\mathbf{c}, \mathbf{d})$ - same with $(\mathbf{a}, \mathbf{b})$, but in presence of hydrogen ions.

In the regime of counterstreaming beams, the highest efficiency is achieved for the collision of thin identical beams with the total injection power $2 P_{1}=7.33 \mathrm{GW}$ (Figure 16a). The peak efficiency of the second harmonic emission in this case reaches $1.4 \%$ (103 MW), $0.6 \%(44 \mathrm{MW})$ goes to the third harmonic and $0.3 \%(22 \mathrm{MW})$ goes to the forth one. In the case of beams with different focal spot sizes (Figure 16b), emission at the third harmonic with the conversion efficiency of up to $\eta \approx 0.5 \%(55 \mathrm{MW})$ dominates. Transition to plasmas with light ions such as hydrogen results in a significant decrease of emission at the third and forth harmonics and most of radiation is generated at the second harmonic due to the head-on collision of different-size waves (Figure 16c,d).

\section{Discussion \& Conclusions}

Recent studies have shown that a thin beam-plasma system (with dimensions comparable to the radiation wavelength) can convert a significant fraction (up to 5-10\%) of the injected beams power into radiation power at harmonics of the plasma frequency due to the mechanisms of the plasma antenna and head-on collision of plasma waves with different potential profiles. In this work, using 2D3V PIC simulations in axially symmetric geometry we have shown how efficiently these mechanisms work for specific parameters of LIA developed in the Budker Institute. The key property of this LIA is that a small enough beam emittance is achieved in such an accelerator at relatively small energies $2 \mathrm{MeV}$ and relatively high currents $1.5-2 \mathrm{kA}$. Such a small emittance allows to focus an electron beam into a spot of $1 \mathrm{~mm}$ diameter which is comparable with the wavelength of EM waves at harmonics of the plasma frequency (lying in $\mathrm{THz}$ range for the typical plasma density $10^{15} \mathrm{~cm}^{-3}$ ) and suited for switching on highly-efficient radiation mechanisms in plasma. High currents corresponding to the beam-to-plasma density ratio $n_{b} / n_{p}=10^{-3}-10^{-2}$ are needed for efficient development of the two-stream instability. The energy of beam particles should be weakly relativistic (1-2 MeV) in order to excite longitudinally propagating plasma waves capable of participating in the discussed emission processes. Moreover, a relatively low energy of beam particles allows to shorten the distance of beam-plasma interaction to the mm-scale making it possible to realize these radiation schemes in gas jets. An increase in energy will lead to an increase in the beam relaxation length (since the growth rate of the two-stream instability is inversely proportional to the relativistic factor) making it longer than the focusing region. Moreover, at high relativistic energies, the spectrum of unstable waves ceases to be dominated by longitudinally propagating modes and one should look for other efficient radiation mechanisms such as three-wave interaction discussed in [31].

PIC modeling of a single beam injection into a plasma without a magnetic field showed that the beam-plasma instability developing near the focus of the beam leads to effective beam bunching with the period of the most unstable wave (several $c / \omega_{p}$ ), while this wave itself (being subluminal) cannot be converted into an electromagnetic one. The possibility of such a conversion opens up if the longitudinal density modulation appears in the plasma 
with a period comparable to the length of the dominant beam-driven wave. However, in the absence of a magnetic field, the radial displacement of plasma from the beam region under the action of the ponderomotive force of plasma oscillations turns out to be a faster process than axial modulation, which does not allow efficient use of the plasma antenna mechanism in this case. Nevertheless, the phenomenon of deep beam bunching near its focus can be used to generate $\mathrm{THz}$ radiation due to the Smith-Purcell mechanism [40,41] or coherent diffraction and transition radiation. A short plasma section (e.g., a supersonic gas jet) in which a periodic sequence of bunches is formed can be used for this purpose. Then these bunches passes through a hole in a metal screen or falls on a metal foil and generate radiation [42]. Switching on a strong magnetic field prevents the radial displacement of the plasma from the beam region, while the growing longitudinal modulation of the plasma density leads to a significant increase in the radiation power at the plasma frequency. The maximum efficiency of such radiation reaches $1.6 \%$.

Another possibility of efficient $\mathrm{THz}$ generation is associated with the use of two colliding LIA beams. In addition to the nonlinear interaction of the most unstable modes $\left(\omega_{0}, \pm k_{0}\right)$, leading to the generation of radiation at the second harmonic, it becomes possible in such a system to generate superluminal oscillations at the third $\left(3 \omega_{0}, \pm k_{0}\right)$ and the fourth $\left(4 \omega_{0}, \pm 2 k_{0}\right)$ harmonics of the plasma frequency. The maximum efficiency of the second harmonic radiation that is achieved by switching on the mechanism of head-on collision of waves with locally different transverse structures, is $1.4 \%$, which in absolute values corresponds to a power of $100 \mathrm{MW}$. The maximum amplitude of the radiation field in this case reaches $\widehat{E}_{z} \approx 10^{-2}$, which corresponds to the value of $340 \mathrm{kV} / \mathrm{cm}$ for the plasma density $n_{0} \approx 1.2 \times 10^{15} \mathrm{~cm}^{-3}$. The most promising development of this scheme is the use of an external magnetic field. This will allow the beams to maintain a given transverse size at a large enough distance. In this case, the use of different-size beams will make it possible to generate radiation near the second harmonic of the plasma frequency at a greater distance and for a longer time, which will significantly increase the efficiency of the scheme.

The duration of radiation in two-beam schemes is limited by effects of ion dynamics. For the plasma density $1.25 \times 10^{15} \mathrm{~cm}^{-3}$, this duration is fractions of a nanosecond. With an increase in the mass of ions (in this work, we considered only the lightest hydrogen ions and infinitely heavy ions), the duration of the radiation increases. In the case of a single beam injection into a magnetic field (Section 3.1), ion dynamics is a necessary condition for switching on the mechanism of the beam-plasma antenna. At much lower plasma densities, this mechanism can operate at much longer times (see experimental results from [24] and simulations of such experiments in [39]).

Compared to other $\mathrm{THz}$ sources based on the use of highly relativistic electron beams [43], plasma-based sources at lower beam energies are attractive for several reasons. The first advantage is the engineering simplicity. In addition to the particle accelerator, only the plasma section is needed, which in fact is a simple gas cell or supersonic gas jet. Second, it is possible to tune radiation frequency within a wide range by a simple changing of the plasma density. Third, in such a scheme it is possible to achieve a THz spectrum width of the order of one percent or less with a high electric field of the order of $\mathrm{MV} / \mathrm{cm}$.

The methods developed in this work for simulating the interaction of relativistic electron beams with plasma are relevant not only for studying promising sources of $\mathrm{THz}$ radiation. The need to investigate this kind of beam-plasma systems also arises in the development of modern X-ray radiography systems based on linear induction accelerators $[44,45]$.

Author Contributions: Conceptualization, I.T.; Data curation, E.B. and E.V.; Formal analysis, V.A.; Funding acquisition, E.B.; Investigation, E.B. and E.V.; Methodology, V.A., E.B. and I.T.; Project administration, I.T.; Resources, E.B.; Software, E.B. and E.V.; Validation, V.A. and I.T.; Visualization, V.A.; Writing—original draft, V.A. and E.B.; Writing—review \& editing, E.V. and I.T. All authors have read and agreed to the published version of the manuscript. 
Funding: The development of the numerical model was supported by the Russian Scientific Foundation (Grant No. 19-71-00054).

Institutional Review Board Statement: Not applicable.

Data Availability Statement: All data included in this study are available upon request by contact with the corresponding author. The code used is available at https://bitbucket.org/berendeev/ luthien_rz, accessed on 1 May 2021.

Acknowledgments: All simulations were performed on HPC-clusters: “Akademik V.M. Matrosov" [46] cluster of NSU [47], NKS-1P SSC [48].

Conflicts of Interest: The authors declare no conflict of interest.

\section{Abbreviations}

The following abbreviations are used in this manuscript:

BINP Budker Institute of Nuclear Physics

SB RAS Siberian Branch of the Russian Academy of Sciences

NSU Novosibirsk State University

FDTD finite-difference time-domain

FEL Free Electron Laser

$\mathrm{THz}$ Teraherz

PIC Particle-in-cell

\section{Appendix A}
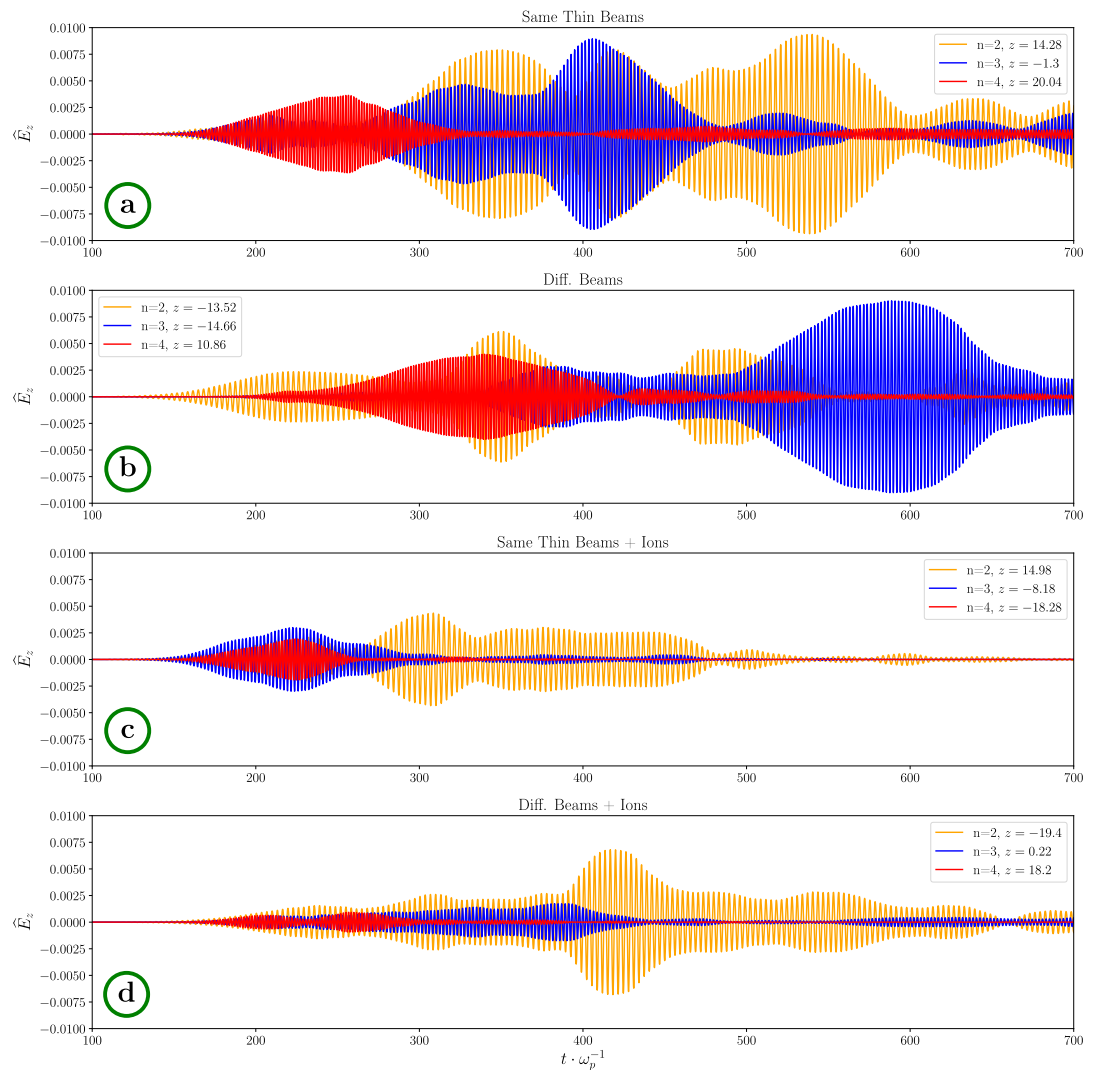

Figure A1. Dependence of the field $\widehat{E}_{z}$ on time for each harmonic of emission at points on the boundary of the computational domain. To construct these curves for each harmonic, the coordinate of the maximum field $\left(z_{\max }, t_{\max }\right)$ was found. Then the time dependence $\widehat{E}_{z}\left(z=z_{\max }, t\right)$ was plotted. (a) same beams with $\sigma_{1}=\sigma_{2}=3.325 \mathrm{c} / \omega_{p} ;(\mathbf{b})$ different beams with $\sigma_{1}=3.325 \mathrm{c} / \omega_{p}$ and $\sigma_{2}=6.65 c / \omega_{p} ;(\mathbf{c}, \mathbf{d})$ - same with $(\mathbf{a}, \mathbf{b})$, but in presence of hydrogen ions. 


\section{References}

1. Dhillon, S.S.; Vitiello, M.S.; Linfield, E.H.; Davies, A.G.; Hoffmann, M.C.; Booske, J.; Paoloni, C.; Gensch, M.; Weightman, P.; Williams, G.P.; et al. The 2017 terahertz science and technology roadmap. J. Phys. D Appl. Phys. 2017, 50, 043001. [CrossRef]

2. Watanabe, S. Terahertz Polarization Imaging and Its Applications. Photonics 2018, 5, 58. [CrossRef]

3. FELIX Laboratory. Available online: http://www.ru.nl/felix/ (accessed on 1 May 2021).

4. Vinokurov, N.A.; Shevchenko, O.A. Free electron lasers and their development at the Budker Institute of Nuclear Physics, SB RAS. Phys. Uspekhi 2018, 61, 435-448. [CrossRef]

5. Kawase, K.; Nagai, M.; Furukawa, K.; Fujimoto, M.; Kato, R.; Honda, Y.; Isoyama, G. Extremely high-intensity operation of a $\mathrm{THz}$ free-electron laser using an electron beam with a higher bunch charge. Nucl. Instrum. Methods Phys. Res. Sect. A Accel. Spectrometers Detect. Assoc. Equip. 2020, 960, 163582. [CrossRef]

6. Liu, B.; Bromberger, H.; Cartella, A.; Gebert, T.; Först, M.; Cavalleri, A. Generation of narrowband, high-intensity, carrier-envelope phase-stable pulses tunable between 4 and $18 \mathrm{THz}$. Opt. Lett. 2017, 42, 129. [CrossRef]

7. Carrillo-Fuentes, M.; Cudney, R.S.; Lee, S.H.; Kwon, O.P. Sub-nanosecond terahertz radiation obtained with an aperiodically poled lithium niobate and organic HMQ-TMS. Opt. Express 2020, 28, 24444. [CrossRef] [PubMed]

8. Vicario, C.; Trisorio, A.; Allenspach, S.; Rüegg, C.; Giorgianni, F. Narrow-band and tunable intense terahertz pulses for mode-selective coherent phonon excitation. Appl. Phys. Lett. 2020, 117, 101101. [CrossRef]

9. Zhang, L.; Cai, J.; Bian, X.; Wu, X.; Feng, J. A Novel THz Forward and Backward Wave Two-Mode Band-Edge Oscillator. IEEE Trans. Terahertz Sci. Technol. 2020, 10, 391-396. [CrossRef]

10. Glyavin, M.Y.; Denisov, G.G.; Zapevalov, V.E.; Koshelev, M.A.; Tretyakov, M.Y.; Tsvetkov, A.I. High power terahertz sources for spectroscopy and material diagnostics. Phys. Uspekhi 2016, 59, 595-604. [CrossRef]

11. Idehara, T.; Sabchevski, S.P.; Glyavin, M.; Mitsudo, S. The Gyrotrons as Promising Radiation Sources for THz Sensing and Imaging. Appl. Sci. 2020, 10, 980. [CrossRef]

12. Thumm, M. State-of-the-Art of High-Power Gyro-Devices and Free Electron Masers. J. Infrared Millim. Terahertz Waves 2020, 41,1-140. [CrossRef]

13. Sheng, Z.M.; Mima, K.; Zhang, J.; Sanuki, H. Emission of Electromagnetic Pulses from Laser Wakefields through Linear Mode Conversion. Phys. Rev. Lett. 2005, 94, 095003. [CrossRef]

14. Miao, C.; Palastro, J.P.; Antonsen, T.M. High-power tunable laser driven THz generation in corrugated plasma waveguides. Phys. Plasmas 2017, 24, 043109. [CrossRef]

15. Timofeev, I.V.; Annenkov, V.V.; Volchok, E.P. Generation of high-field narrowband terahertz radiation by counterpropagating plasma wakefields. Phys. Plasmas 2017, 24, 103106. [CrossRef]

16. Kwon, K.B.; Kang, T.; Song, H.S.; Kim, Y.K.; Ersfeld, B.; Jaroszynski, D.A.; Hur, M.S. High-Energy, Short-Duration Bursts of Coherent Terahertz Radiation from an Embedded Plasma Dipole. Sci. Rep. 2018, 8, 145. [CrossRef] [PubMed]

17. Ushakov, A.; Chizhov, P.; Bukin, V.; Shipilo, D.; Panov, N.; Kosareva, O.; Garnov, S. Multiple Filamentation Effects on THz Radiation Pattern from Laser Plasma in Air. Photonics 2020, 8, 4. [CrossRef]

18. Bogatskaya, A.V.; Gnezdovskaia, N.E.; Popov, A.M. Tunable THz pulses generation in non-equilibrium magnetized plasma: The role of plasma kinetics. Photonics 2020, 7, 82. [CrossRef]

19. Kalmykov, S.; Elle, J.; Schmitt-Sody, A. Radiation emission at Langmuir frequency from laser wake in longitudinally stratified plasma column. Plasma Phys. Control. Fusion 2020, 62, 115022. [CrossRef]

20. Arzhannikov, A.V.; Ginzburg, N.S.; Zaslavsky, V.Y.; Kalinin, P.V.; Peskov, N.Y.; Sandalov, E.S.; Sergeev, A.S.; Sinitsky, S.L.; Stepanov, V.D. Planar THz FELs Based on Intense Parallel Sheet Electron Beams and Intracavity Wave Scattering. Bull. Russ. Acad. Sci. Phys. 2019, 83, 140-145. [CrossRef]

21. Peskov, N.Y.; Abubakirov, E.B.; Arzhannikov, A.V.; Denisenko, A.N.; Ginzburg, N.S.; Malkin, A.M.; Kalinin, P.V.; Sandalov, E.S.; Sinitsky, S.L.; Stepanov, V.D.; et al. Powerful Cherenkov masers with 2D slow-wave structures intended for powering systems of Compton-type FELs. In Fourth International Conference on Terahertz and Microwave Radiation: Generation, Detection, and Applications; Romanovskii, O.A., Kistenev, Y.V., Eds.; SPIE: Bellingham, WA, USA, 2020; p. 10. [CrossRef]

22. Peskov, N.Y.; Ginzburg, N.S.; Golubev, I.I.; Golubykh, S.M.; Kaminsky, A.K.; Kozlov, A.P.; Malkin, A.M.; Sedykh, S.N.; Sergeev, A.S.; Sidorov, A.I.; et al. Powerful oversized W-band free-electron maser with advanced Bragg resonator based on coupling of propagating and cutoff waves. Appl. Phys. Lett. 2020, 116, 213505. [CrossRef]

23. Peskov, N.Y.; Ginzburg, N.S.; Zaslavsky, V.Y.; Arzhannikov, A.V.; Kalinin, P.V.; Sinitsky, S.L. Powerful Long-Pulse Bragg FEL of Sub-THz to THz-band: Simulations and Tests of Electrodynamic System. In Proceedings of the 2020 7th All-Russian Microwave Conference (RMC), Moscow, Russia, 25-27 November 2020; pp. 74-76. [CrossRef]

24. Burdakov, A.V.; Arzhannikov, A.V.; Burmasov, V.S.; Ivanov, I.A.; Ivantsivsky, M.V.; Kandaurov, I.V.; Kuznetsov, S.A.; Kurkuchekov, V.V.; Mekler, K.I.; Polosatkin, S.V.; et al. Microwave Generation During 100 keV Electron Beam Relaxation in GOL-3. Fusion Sci. Technol. 2013, 63, 286-288. [CrossRef]

25. Timofeev, I.V.; Annenkov, V.V.; Arzhannikov, A.V. Regimes of enhanced electromagnetic emission in beam-plasma interactions. Phys. Plasmas 2015, 22, 113109. [CrossRef]

26. Timofeev, I.V.; Volchok, E.P.; Annenkov, V.V. Theory of a beam-driven plasma antenna. Phys. Plasmas 2016, 23, 083119. [CrossRef]

27. Annenkov, V.V.; Volchok, E.P.; Timofeev, I.V. Generation of high-power electromagnetic radiation by a beam-driven plasma antenna. Plasma Phys. Control. Fusion 2016, 58, 045009. [CrossRef] 
28. Timofeev, I.V.; Berendeev, E.A.; Dudnikova, G.I. Simulations of a beam-driven plasma antenna in the regime of plasma transparency. Phys. Plasmas 2017, 24, 093114. [CrossRef]

29. Arzhannikov, A.V.; Ivanov, I.A.; Kasatov, A.A.; Kuznetsov, S.A.; Makarov, M.A.; Mekler, K.I.; Polosatkin, S.V.; Popov, S.S.; Rovenskikh, A.F.; Samtsov, D.A.; et al. Well-directed flux of megawatt sub-mm radiation generated by a relativistic electron beam in a magnetized plasma with strong density gradients. Plasma Phys. Control. Fusion 2020, 62, 045002. [CrossRef]

30. Annenkov, V.V.; Berendeev, E.A.; Timofeev, I.V.; Volchok, E.P. High-power terahertz emission from a plasma penetrated by counterstreaming different-size electron beams. Phys. Plasmas 2018, 25, 113110. [CrossRef]

31. Annenkov, V.V.; Volchok, E.P.; Timofeev, I.V. Electromagnetic Emission Produced by Three-wave Interactions in a Plasma with Continuously Injected Counterstreaming Electron Beams. Astrophys. J. 2020, 904, 88. [CrossRef]

32. Logachev, P.V.; Kuznetsov, G.I.; Korepanov, A.A.; Akimov, A.V.; Shiyankov, S.V.; Pavlov, O.A.; Starostenko, D.A.; Fat'Kin, G.A. LIU-2 linear induction accelerator. Instrum. Exp. Tech. 2013, 56, 672-679. [CrossRef]

33. Boris, J.P. Relativistic plasma simulation-optimization of a hybrid code. In Proceeding of Fourth Conference on Numerical Simulations of Plasmas; Naval Research Laboratory: Washington, DC, USA, 1970; pp. 3-67.

34. Esirkepov, T. Exact charge conservation scheme for Particle-in-Cell simulation with an arbitrary form-factor. Comput. Phys. Commun. 2001, 135, 144-153. [CrossRef]

35. Taflove, A.; Hagness, S.C.; Piket-May, M. Computational Electromagnetics: The Finite-Difference Time-Domain Method. In The Electrical Engineering Handbook; Elsevier: Amsterdam, The Netherlands, 2005; pp. 629-670. [CrossRef]

36. Annenkov, V.V.; Timofeev, I.V.; Volchok, E.P. Simulations of electromagnetic emissions produced in a thin plasma by a continuously injected electron beam. Phys. Plasmas 2016, 23, 053101. [CrossRef]

37. Berenger, J.P. A perfectly matched layer for the absorption of electromagnetic waves. J. Comput. Phys. 1994, 114, 185-200. [CrossRef]

38. Annenkov, V.V.; Berendeev, E.A.; Volchok, E.P.; Timofeev, I.V. Second harmonic electromagnetic emission in a beam-driven plasma antenna. Plasma Phys. Control. Fusion 2019, 61, 055005. [CrossRef]

39. Annenkov, V.V.; Timofeev, I.V.; Volchok, E.P. Highly efficient electromagnetic emission during 100 keV electron beam relaxation in a thin magnetized plasma. Phys. Plasmas 2019, 26, 063104. [CrossRef]

40. Smith, S.J.; Purcell, E.M. Visible light from localized surface charges moving across a grating. Phys. Rev. 1953, 92, 1069. [CrossRef]

41. Zhang, H.; Konoplev, I.; Doucas, G. A tunable source of coherent terahertz radiation driven by the microbunched electron beam. J. Phys. D Appl. Phys. 2020, 53, 105501. [CrossRef]

42. Jackson, J.D. Classical Electrodynamics, 3rd ed.; Wiley \& Sons Ltd.: Hoboken, NJ, USA, 1998; p. 808.

43. Chiadroni, E.; Cianchi, A.; Ferrario, M.; Mostacci, A.; Pompili, R.; Shpakov, V. A Versatile THz Source from High-Brightness Electron Beams: Generation and Characterization. Condens. Matter 2020, 5, 40. [CrossRef]

44. Zhu, J.; Yu, H.; Jiang, X.; Cheng, N.; Wang, Y. Target-plasma expansion induced by 20-MeV intense electron beam. IEEE Trans. Plasma Sci. 2010, 38, 2873-2876. [CrossRef]

45. Trunev, Y.A.; Skovorodin, D.I.; Burdakov, A.V.; Popov, S.S.; Kolesnikov, P.A.; Danilov, V.V.; Kurkuchekov, V.V.; Atlukhanov, M.G.; Kulenko, I.V.; Arakcheev, A.S.; et al. Observation of the Dynamics of a Focal Spot Using a Long-Pulse Linear Induction Accelerator. IEEE Trans. Plasma Sci. 2020, 48, 2125-2131. [CrossRef]

46. Irkutsk Supercomputer Center of SB RAS. Available online: http:/ / hpc.icc.ru (accessed on 1 May 2021).

47. Novosibirsk University Scientific Computing Facility. Available online: http:/ / nusc.nsu.ru (accessed on 1 May 2021).

48. Siberian Supercomputer Center of SB RAS. Available online: http://www.sscc.icmmg.nsc.ru/ (accessed on 1 May 2021). 